\title{
A Performance Indicator for Reference-Point-Based Multiobjective Evolutionary Optimization
}

\author{
$1^{\text {st }}$ Zhanglu Hou \\ Key Laboratory of Intelligent Computing \\ and Information Processing (Ministry of Education) \\ Xiangtan 411105, China \\ Email: zhanglhou@163.com
}

\author{
$2^{\text {nd }}$ Shengxiang Yang \\ School of Computer Science and Informatics \\ De Montfort University \\ Leicester LE1 9BH,U.K. \\ Email: syang@dmu.ac.uk
}

\author{
$3^{\text {rd }}$ Juan Zou (* Corresponding author) \\ Key Laboratory of Intelligent Computing \\ and Information Processing (Ministry of Education) \\ Xiangtan 411105, China \\ Email: zoujuan@xtu.edu.cn
}

\author{
$5^{\text {th }} \mathrm{Guo} \mathrm{Yu}$ \\ Department of Computer Science \\ University of Surrey \\ Guildford,Surrey,GU2 7XH,UK \\ Email: guo.yu@surrey.ac.uk
}

\author{
$6^{\text {th }}$ Gan Ruan \\ Key Laboratory of Intelligent Computing \\ and Information Processing (Ministry of Education)) \\ Xiangtan 411105, China \\ Email: ruangan199332@gmail.com
}

\begin{abstract}
Aiming at the difficulty in evaluating preferencebased evolutionary multiobjective optimization, this paper proposes a new performance indicator. The main idea is to project the preferred solutions onto a constructed hyperplane which is perpendicular to the vector from the reference (aspiration) point to the origin. And then the distance from preferred solutions to the origin and the standard deviation of distance from each mapping point to the nearest point will be calculated. The former is used to measure the convergence of the obtained solutions. The latter is utilized to assess the diversity of preferred solutions in the region of interest. The indicator is conducted to assess different algorithms on a series of benchmark problems with various features. The results show that the proposed indicator is able to properly evaluate the performance of preference-based multiobjective evolutionary algorithms.
\end{abstract}

Index Terms-reference point, preference, indicator

\section{INTRODUCTION}

As most real-life decisions and problems involve multiple conflicting criteria that must be considered simultaneously, multiobjective evolutionary algorithms (MOEAs) have been widely adopted to deal with multiobjective optimization problems [1], [2], [3], [4], [5]. However, it is difficult for the decision maker (DM) to select the final preferred solutions among the whole Pareto-optimal front (POF).

Thus, preference-based MOEAs [6], [7], [8] have been developed to facilitate the DM by providing solutions in the regions of interest (ROIs) according to DM's preference information. Notably, there are two advantages embedding the preference information from the DM into the MOEAs. First, in terms of the preference information, MOEAs could save more evaluations or computational resources during environmental selection so as to focus on searching solutions in the ROIs [9] rather than on the solutions approximating to the whole POF. Second, a number of preferred solutions could aid the DM to make a better decision.

Although a series of preference-based MOEAs [10], [11], [12] have been proposed, the performance metrics are still few and should be improved. To facilitate the DM, preferencebased metrics should reflect different performances of the algorithms that have been compared by evaluating the provided solution sets but also need to meet the DM's preferences. Traditional performance metrics such as [13] , [14] are based on the POF of benchmark problems. However, in most cases, we have no idea about the POF [15] in engineering applications. It is of great practical significance to develop the preference-based indicators that do not require the information of true POF. Recently, Mohammeadi et al. [16] proposed a performance metric (IGD-CF) by combining a composite POF and classical MOEA assessment for comparing different preference-based MOEAs. This metric is effective when the composite front is fairly close to the POF and at the lower dimensional space. Moreover, Li et al. [17] put forward a new performance metric (R-metric), whose basic idea is to preprocess the approximation sets found by different algorithms before using the inverted generational distance (IGD) [14]. However, in this metric, the POF is also supposed to be known and it can not assess all preferred solutions obtained by a preference-based MOEA. 
In this paper, we design a indicator to assess the preferencebased MOEAs using a reference point. The main idea is to project preferred solutions onto a hyperplane which is perpendicular to the vector connecting preference point and origin, and then calculating some specific distances to assess the convergence and diversity of preferred solutions. This indicator does not need any information about the POF and it can help the DM make a better decision via the evaluation mechanism.

The rest of this paper is organized as follows. Section II briefly introduces various existing performance metrics. Section III explains the details of the proposed performance indicator. Section IV verifies the effectiveness of the proposed method and analyses the experimental results. Finally conclusions are drawn.

\section{BACKGROUND}

In this section, several quality metrics are introduced to evaluate the performance of preference-based multiobjective evolutionary algorithms.

\section{A. Generational Distance}

Generational distance (GD) [13] is widely applied to measure the convergence of the approximated set $\mathrm{P}$ towards the POF. The GD is defined as:

$$
G D=\frac{\sum d(i, P O F)}{|P|}, i \in P
$$

where $|P|$ is the number of solutions in $\mathrm{P}$, and $\mathrm{d}(\mathrm{i}, \mathrm{POF})$ is the nearest Euclidean distance between each solution $\mathrm{i}$ and the POF. The GD still has some shortcomings although it can assess the convergence of preference-based MOEAs effectively. On the one hand, it requires the knowledge of the whole POF. In addition, it has to sample a large set of uniformly distributed points on the POF, which is hard to be precisely achieved in many-objective problems. On the other hand, GD will be misleading when dealing with preferencebased problems. Since the obtained solutions, far from the ROI, might get a better GD value.

\section{B. $I G D-C F$}

Inverted Generational Distance Based Composite Front (IGD-CF) [16] is a comprehensive performance metric which can measure both the convergence and diversity of the obtained solution set. IGD-CF substitutes a composite front for the POF and then defines the ROI by means of a user-supplied reference point. Finally it calculates the IGD value of solutions within the region of interests. Fig.1 shows an example of how to define a preferred region by a composite front.

The metric does not require any prior knowledge of the POF and takes the DM's preference information into consideration. Nonetheless, there are some flaws in IGD-CF. It will give wrong information when the composite front is not fairly close and similar to the POF. Moreover, as described in [17], the objectives need to be normalized, which may be a drawback. Besides, the parameter $r$ shown in Fig.1 will not be meaningful because real-world problems do not

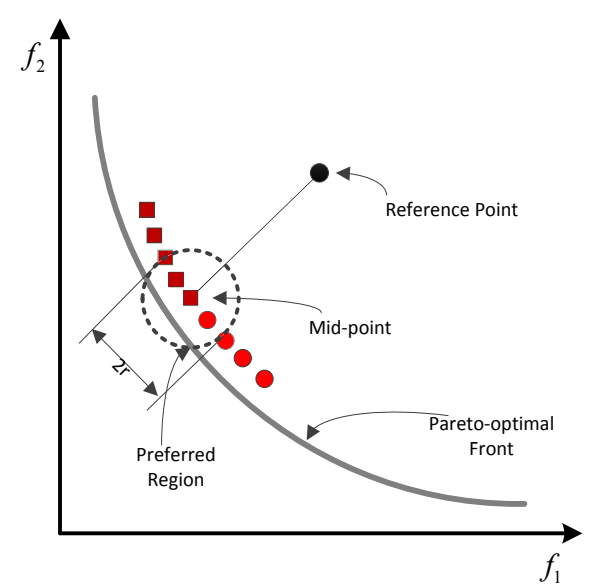

Fig. 1. Illustration of a composite front. The composite front is composed of the red circles and squares.

have the same units.

\section{R-metric}

R-metric [17] is a metric which measures both the diversity and convergence of preferred solutions. It provides a unified framework for performance assessment of a partial POF. The method is used first to remove the dominated solutions, then to translate the filtered solutions to a virtual position along the iso-ASF line shown in Fig.2. Last, the baseline metric IGD or other metrics are chosen to evaluate the quality of a preferred efficient set. The basic outline of the algorithm is given as:

- Prescreen procedure

Assume that there are L preferred efficient sets which are obtained by L different preference-based MOEAs. Before the assessment of the preferred efficient sets, the metric needs to prescreen them and only the non-dominated solutions can be used for R-metric computation.

- Center identification Use the solution closest to the centroid of the underlying solution set as the representative point.

- Filtering procedure

Keep the solutions, which are close to the representative point and within a relative extent of the ROI, for the Rmetric calculation.

- Solution transfer

Translate the filtered solutions towards the reference line constructed by the reference point and a given worst point. Then, the regular metrics IGD can be applied for performance assessment.

R-metric does not assess all preferred solutions given by the preference-based MOEA using a reference point. In other words, the value of the metric cannot reflect back the true 


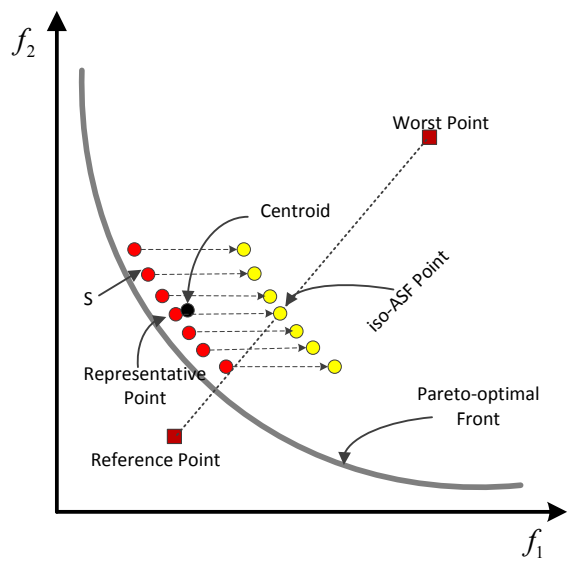

Fig. 2. Illustration of the R-metric computation procedure

performance of preference-based MOEA, which may mislead the DM. Furthermore, it will burden DM to provide an additional worst point in the R-IGD computation. In addition, the metric, similar to the original IGD, needs to know the POF.

\section{A PROPOSED PERFORMANCE INDICATOR}

In this section, a novel reference-point-based performance indicator is proposed to evaluate the convergence and diversity of preference-based MOEAs, and the indicator is termed PMOD for convenience. Different from other metrics, the proposed indicator maps the obtained solutions to a constructed hyperplane integrating with preference information, then different distances are calculated to evaluate different aspects of the solutions. The details are as follows.

Step 1: Construct the hyperplane and define a preferred region.

Given a reference point from the DM, it define a normal vector which is constituted from reference point to the origin of coordinate or a direction vector $w$ implying relative importance of each objective, then the preference-based hyperplane $S$ can be formulated according to the reference point and normal vector. For example, assuming a reference point $R(a, b, c)$, the hyperplane can be defined as $a(f 1-a)+b(f 2-b)+c(f 3-c)=$ 0 . The reference point $R$ is selected to be center point of the ROI, and the size of preferred region can be adjusted by the decision maker with a Euclidean distance $r$ from center point.

\section{Step 2: Obtain the mapping points.}

Make a vector $\overrightarrow{p R}$ from reference point $\mathrm{R}$ to the solution $\mathrm{p}$, $\overrightarrow{P R}=\overrightarrow{O R}-\overrightarrow{O P}$. Then take the dot product of that vector with the unit normal vector, $\vec{n}=\frac{\overrightarrow{O R}}{|\overrightarrow{O R}|}$, dist $=\overrightarrow{p R} \cdot \vec{n}$. Finally, multiply the unit normal vector by the distance, and add that vector from preferred point, $\overrightarrow{O p^{\prime}}=\overrightarrow{O p}+\overrightarrow{p p^{\prime}}=\overrightarrow{O p}+\operatorname{dist} * \vec{n}$, mapping point p' $\in \mathrm{S}$ shown in Fig.3.

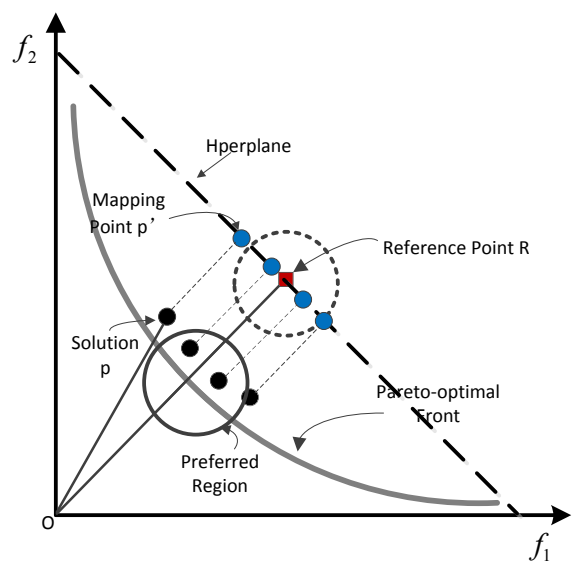

Fig. 3. Illustration of the PMOD on two-dimensional space

Step 3: Specific distances computation.

Calculate distance $D 1$ between the mapping point and reference point and the standard deviation of distance $D 2$ from each mapping point to the nearest point. For the preferred set $P^{\prime}$ of mapping points, $D 2$ can be calculated as follows:

$$
\sqrt{\frac{1}{\left|P^{\prime}\right|-1} \sum_{i=1}^{\left|P^{\prime}\right|}\left(\bar{d}-d_{i}\right)^{2}}
$$

where $d_{i}=\min _{q_{j} \in P^{\prime} \wedge q_{j} \neq q_{i}} \sum_{m=1}^{M}\left|f_{m}\left(q_{i}\right)-f_{m}\left(q_{j}\right)\right| ; \bar{d}$ is the mean of all $d_{i}$, and $M$ is the number of objectives. Then, we compute the Euclidean distance $D 3$ between preferred solution and origin. If the mapping point of the preferred solution is not in the ROI, multiply the distance by a penalty coefficient $k$. For the solution $p$ shown in Fig. $3, D 1=\left|\overrightarrow{p^{\prime} R}\right|, D 3=|\overrightarrow{O p}|$.

Step 4: Calculate the performance metric value. The metric can be defined:

$$
P M O D=\frac{\sum_{1}^{n}(D 1+k D 3)}{n}+D 2
$$

where

$$
\begin{cases}k=1 & r \leq D 1 \\ k>1 & \text { otherwise }\end{cases}
$$

$n$ is the number of the preferred solutions.

As can be seen from equation (3), the smaller the value of PMOD, the better performance of the algorithm. The advantage of PMOD is that it does not require knowledge of the POF and can measure both the convergence and diversity by simple distances calculation. Moreover, the proposed indicator does not need to normalize the objective space when determining the size of the interest of region. As described in IGD-CF and R-metric, the setting of ROI's size assumes the objective space is normalized to $[0,1]$, but this assumption might not always hold in real problems [2]. 


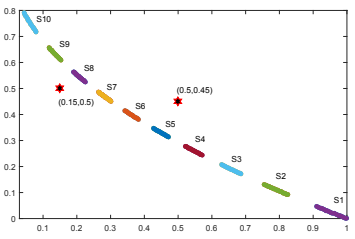

(a)population distribution

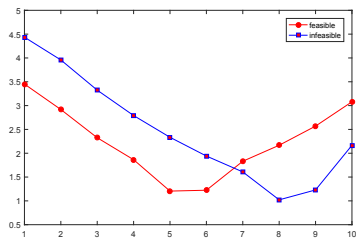

(b)the value of PMOD
Fig. 4. Reference point $(0.15,0.5)$ in infeasible region and reference point $(0.5,0.45)$ in feasible region on ZDT1 problem

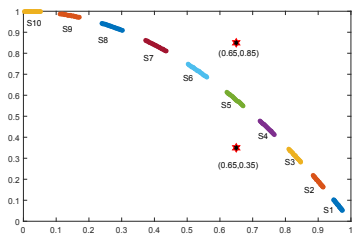

(a)population distribution

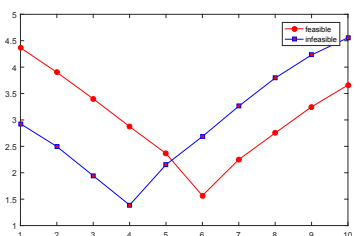

(b)the value of PMOD
Fig. 5. Reference point $(0.65,0.35)$ in infeasible region and reference point $(0.65,0.85)$ in feasible region on ZDT2 problem

\section{EXPERIMENTAL ANALYSIS}

In this section, to verify the effectiveness of the proposed indicator objectively, we employ the method suggested in Rmetric [17]. A good indicator can deal with different shapes including the convex and non-convex POF as well as different locations of the reference point; thus two-objective benchmark problems ZDT1 and ZDT2 [19] are applied. We sampled ten sets of points, $\mathrm{S} 1$ to $\mathrm{S} 10$, from different regions of the POF. Each set has 25 points distributing relatively evenly; the penalty coefficient $k$ and the parameter $r$ are set as 1.5 and 0.1 respectively. The settings of reference point in the infeasible and feasible region are shown in Table I.

TABLE I

REFERENCE POINT SETTING FOR ZDT1 AND ZDT2

\begin{tabular}{c|c|c}
\hline Benchmark problem & Infeasible region & Feasible region \\
\hline ZDT1 & $(0.15,0.5)$ & $(0.5,0.45)$ \\
\hline ZDT2 & $(0.65,0.35)$ & $(0.65,0.85)$ \\
\hline
\end{tabular}

From the results shown in Fig.4 and Fig.5, the indicator value makes a reasonable assessment on the quality of a point set in accordance with the DM's preference information. For example, the PMOD value of S4 is indeed the best in Fig.5(b) since it is closest to the ROI with respect to reference point $(0.65,0.35)$. For the other point sets, the farther away from the ROI, the worse the indicator values.

In the following, we apply the proposed indicator to evaluate the performance of two state-of-art preference-based MOEAs g-NSGA-II [6] and r-NSGA-II [7]. The population size is set to 100 for both 2-objective and 3-objective problems and 200 for many-objective problems. ZDT1-ZDT4 and ZDT6 [19] are chosen to be the 2-objective test problems. DTLZ1DTLZ4 and DTLZ6 [20] are chosen to the 3-objective test problems, and DTLZ2 is used as the many-objective problem with 5-,8- and 10-objectives. The maximum number of the generation is 500 on ZDT test problems and 1000 on DTLZ test problems. The preference-based algorithms have been run 30 times independently on each problem. Table II and Table III give the reference point setting for test problems. Two different penalty coefficients 1.5 and 2.0 are applied in order to test the strength of the penalty.

TABLE II

THE SETTINGS OF REFERENCE POINTS

\begin{tabular}{c|c|c}
\hline Benchmark problem & Infeasible region & Feasible region \\
\hline ZDT1 & $(0.3,0.4)$ & $(0.4,0.6)$ \\
ZDT2 & $(0.2,0.8)$ & $(0.6,0.8)$ \\
ZDT3 & $(0.1,0.2)$ & $(00.4,0.6)$ \\
ZDT4 & $(0.1,0.2)$ & $(0.5,0.5)$ \\
ZDT6 & $(0.1,0.2)$ & $(0.7,0.8)$ \\
\hline DTLZ1 & $(0.1,0.2,0.1)$ & $(0.4,0.8,0.8)$ \\
DTLZ2 & $(0.1,0.2,0.1)$ & $(0.4,0.8,0.8)$ \\
DTLZ3 & $(0.1,0.2,0.1)$ & $(0.4,0.8,0.8)$ \\
DTLZ4 & $(0.1,0.2,0.1)$ & $(0.4,0.8,0.8)$ \\
DTLZ6 & $(0.1,0.2,0.1)$ & $(0.7,0.6,0.6)$ \\
\hline
\end{tabular}

To have a visual comparison and evaluate the effectiveness of the proposed indicator, we plotted the final solutions of several test problems obtained by the algorithms and combine it with the specific data to analyse the performance of preferredbased algorithms.

TABLE III

REFERENCE POINT SETTING FOR MANY-OBJECTIVE PROBLEM DTLZ2

\begin{tabular}{c|c|c|c}
\hline Objective & 5 & 8 & 10 \\
\hline Reference & $(0.1,0.3,0.2$ & $(0.3,0.3,0.3,0.1$ & $(0.3,0.3,0.3,0.1,0.3,0.5$ \\
point & $0.4,0.2)$ & $0.3,0.35,0.35)$ & $0.35,0.35,0.35,0.25,0.45)$ \\
\hline
\end{tabular}

From results of the ZDT test problems, the two preferencebased algorithms do not have too much difficulty in finding convergent solutions around the DM supplied reference point. But shown in Fig.7(a), there are lots of solutions obtained by g-NSGA-II which deviates from the ROI. Accordingly, the value of metric is worse than the value obtained by r-NSGA-II.

According to the figures of the DTLZ test problems, solutions obtained by r-NSGA-II are overall more in line with DM's aspiration. DTLZ2 and DTLZ4 have the same POF shape. From Fig. 8 and Fig.10, it can clearly see that solutions obtained by r-NSGA-II are better than the other algorithms. Although the solutions obtained by g-NSGA-II are convergent, majorities of the solutions are out of ROI. Thus the value of PMOD calculated by g-NSGA-II is worse than r-NSGAII. DTLZ3 has the same POF shape as DTLZ4, but is with $3^{10}-1$ local efficient fronts. From Fig.9, we can see that the g-NSGA-II and r-NSGA-II have difficulty in obtaining satisfactory solutions, g-NSGA-II does not converge at all and the PMOD gives a reasonable value shown in Table IV. 
TABLE IV

THE EXPERIMENTAL RESULTS ON THE SET OF ZDT AND DTLZ WITH TWO LOCATIONS OF REFERENCE POINT.

\begin{tabular}{|c|c|c|c|c|c|}
\hline \multirow{2}{*}{ Metric } & \multirow{2}{*}{ Problem } & \multicolumn{2}{|c|}{ Infeasible Region } & \multicolumn{2}{|c|}{ Feasible Region } \\
\hline & & g-NSGA-II & r-NSGA-II & g-NSGA-II & r-NSAGA-II \\
\hline \multirow{10}{*}{$\begin{array}{l}\text { PMOD } \\
(k=1.5)\end{array}$} & ZDT1 & $1.069 \mathrm{E}+00(5.78 \mathrm{E}-02)$ & $1.036 \mathrm{E}+00(1.53 \mathrm{E}-02)$ & $2.254 \mathrm{E}+00(9.97 \mathrm{E}-03)$ & $1.713 E+00(7.27 E-02)$ \\
\hline & & $2.559 \mathrm{E}+\mathrm{OO}(1.51 \mathrm{E}-01)$ & $1.966 \mathrm{E}+00(6.32 \mathrm{E}-03)$ & $1.855 \mathrm{E}+\mathrm{OO}(\mathbf{7 . 1 4 E - 0 3})$ & $1.728 \mathrm{E}+00(3.78 \mathrm{E}-\mathrm{O} 2)$ \\
\hline & ZDT3 & $2.966 \mathrm{E}+00(3.92 \mathrm{E}-02)$ & $2.969 \mathrm{E}+\mathrm{OO}(1.93 \mathrm{E}-\mathrm{O} 1)$ & $2.729 \mathrm{E}+00(2.96 \mathrm{E}-02)$ & $3.111 \mathrm{E}+00(1.08 \mathrm{E}-01)$ \\
\hline & ZDT4 & $3.789 \mathrm{E}+00(7.49 \mathrm{E}-02)$ & $4.521 \mathrm{E}+\mathrm{OO}(4.51 \mathrm{E}-02)$ & $2.177 \mathrm{E}+00(1.30 \mathrm{E}-02)$ & $2.601 \mathrm{E}+00(1.36 \mathrm{E}-01)$ \\
\hline & ZDT6 & 6.059E $+\mathrm{OO}(\mathbf{2 . 0 4 E - 0 2})$ & $5.969 E+00(4.35 E-02)$ & $2.634 \mathrm{E}+00(1.09 \mathrm{E}-02)$ & $3.365 \mathrm{E}+\mathrm{OO}(4.11 \mathrm{E}-\mathrm{O} 2)$ \\
\hline & DTLZ1 & $1.560 \mathrm{E}+03(3.92 \mathrm{E}+02)$ & $2.822 \mathrm{E}+00(6.65 \mathrm{E}-02)$ & $1.417 \mathrm{E}+03(2.21 \mathrm{E}+02)$ & $7.557 \mathrm{E}+00(7.35 \mathrm{E}-01)$ \\
\hline & DTLZ2 & $7.782 \mathrm{E}+\mathrm{OO}(5.96 \mathrm{E}-\mathrm{O} 1)$ & $6.692 \mathrm{E}+00(4.26 \mathrm{E}-02)$ & $3.545 \mathrm{E}+\mathrm{OO}(\mathbf{3 . 0 7} \mathbf{E - 0 2})$ & 2.167E+00(1.07E-01) \\
\hline & DTLZ3 & $2.746 \mathrm{E}+03(8.96 \mathrm{E}+02)$ & $7.357 \mathrm{E}+00(4.40 \mathrm{E}-01)$ & $2.958 \mathrm{E}+03(6.19 \mathrm{E}+02)$ & $6.348 \mathrm{E}+00(9.96 \mathrm{E}-02)$ \\
\hline & & $9 \mathrm{E}+\mathrm{OO}(3.02 \mathrm{E}-01)$ & $6.723 \mathrm{E}+00(3.79 \mathrm{E}-02)$ & $3.524 \mathrm{E}+\mathrm{OO}(\mathbf{4 . 0 3 E - 0 2})$ & $3.249 \mathrm{E}+00(7.12 \mathrm{E}-\mathrm{O} 2)$ \\
\hline & Z6 & $3.952 \mathrm{E}+01(5.95 \mathrm{E}+00)$ & $7.505 \mathrm{E}+00(2.38 \mathrm{E}-01)$ & $3.756 \mathrm{E}+01(8.55 \mathrm{E}+00)$ & $2.971 \mathrm{E}+00(8.30 \mathrm{E}-02)$ \\
\hline \multirow{10}{*}{$\begin{array}{l}\text { PMOD } \\
(\mathrm{k}=2.0)\end{array}$} & DT1 & $1.122 \mathrm{E}+\mathrm{OO}(1.65 \mathrm{E}-01)$ & $1.030 \mathrm{E}+00(1.48 \mathrm{E}-02)$ & $2.413 \mathrm{E}+\mathrm{OO}(1.56 \mathrm{E}-02)$ & $1.713 E+00(7.27 E-02)$ \\
\hline & & $2.796 \mathrm{E}+\mathrm{OO}(1.50 \mathrm{E}-01)$ & $1.966 \mathrm{E}+00(6.18 \mathrm{E}-03)$ & $1.908 \mathrm{E}+\mathrm{OO}(\mathbf{9 . 4 7 \mathrm { E } - 0 3 )}$ & $1.729 \mathrm{E}+00(3.75 \mathrm{E}-\mathrm{O} 2)$ \\
\hline & DT3 & $3.086 \mathrm{E}+\mathrm{OO}(\mathbf{4 . 1 6 \mathrm { E } - 0 2})$ & $2.970 E+00$ & $2.830 \mathrm{E}+00(3.28 \mathrm{E}-02)$ & $3.122 \mathrm{E}+\mathrm{OO}(1.30 \mathrm{E}-01)$ \\
\hline & ZDT4 & 4.065E+00 $(7.69 \mathrm{E}-02)$ & 4.937E+OO(4.58E-02) & $2.320 \mathrm{E}+00(2.01 \mathrm{E}-02)$ & $2.984 \mathrm{E}+\mathrm{OO}(1.37 \mathrm{E}-01)$ \\
\hline & ZDT6 & $6.425 \mathrm{E}+\mathrm{OO}(2.46 \mathrm{E}-02)$ & 6.108E+00 $(5.57 \mathrm{E}-02)$ & $2.860 \mathrm{E}+00(1.75 \mathrm{E}-02)$ & $3.709 \mathrm{E}+\mathrm{OO}(4.75 \mathrm{E}-\mathrm{O} 2)$ \\
\hline & DTLZ1 & $1.566 \mathrm{E}+03(3.93 \mathrm{E}+02)$ & $3.081 \mathrm{E}+00(4.96 \mathrm{E}-02)$ & $1.423 \mathrm{E}+03(2.22 \mathrm{E}+02)$ & $7.853 \mathrm{E}+00(7.33 \mathrm{E}-01)$ \\
\hline & DTLZ2 & $8.296 \mathrm{E}+00(6.00 \mathrm{E}-01)$ & $7.187 \mathrm{E}+00(5.53 \mathrm{E}-02)$ & $3.993 \mathrm{E}+\mathrm{OO}(\mathbf{4 . 3 0 E - 0 2})$ & $2.239 \mathrm{E}+00(1.56 \mathrm{E}-01)$ \\
\hline & DTLZ3 & $2.754 \mathrm{E}+03(8.98 \mathrm{E}+\mathrm{O} 2)$ & $7.853 \mathrm{E}+00(4.43 \mathrm{E}-01)$ & $2.965 \mathrm{E}+03(6.20 \mathrm{E}+02)$ & $6.805 E+00(9.88 E-02)$ \\
\hline & DTLZ4 & $7.954 \mathrm{E}+00(3.09 \mathrm{E}-01)$ & $7.204 \mathrm{E}+00(5.11 \mathrm{E}-02)$ & 3.956E+OO(4.71E-02) & $\mathbf{3 . 6 6 7 E}+00(8.37 \mathrm{E}-\mathrm{O} 2)$ \\
\hline & DTLZ6 & $3.974 \mathrm{E}+01(7.55 \mathrm{E}+00)$ & $7.960 \mathrm{E}+00(1.85 \mathrm{E}-01)$ & $3.864 \mathrm{E}+01(8.70 \mathrm{E}+00)$ & $3.375 \mathrm{E}+00(9.42 \mathrm{E}-02)$ \\
\hline
\end{tabular}

TABLE V

THE EXPERIMENTAL RESULTS ON DTLZ2 WITH 5-,8-,10-OBJECTIVES.

\begin{tabular}{c|c|c|c|c|c|c}
\hline \multirow{2}{*}{ Metric } & \multicolumn{2}{|c|}{ 5-objective } & \multicolumn{2}{c}{ 8-objective } & \multicolumn{2}{c}{ 10-objective } \\
\cline { 2 - 6 } & g-NSGA-II & r-NSGA-II & g-NSGA-II & r-NSAGA-II & g-NSGA-II & r-NSGA-II \\
\hline PMOD $(k=1.5)$ & $2.516 \mathrm{E}+01(1.09 \mathrm{E}+00)$ & $\mathbf{5 . 7 3 5 E + 0 0 ( 4 . 4 3 E - 0 2 )}$ & $2.793 \mathrm{E}+01(1.91 \mathrm{E}-01)$ & $\mathbf{1 . 7 7 8 E + 0 0 ( 3 . 6 0 E - 0 2 )}$ & $2.786 \mathrm{E}+01(2.35 \mathrm{E}-01)$ & $\mathbf{1 . 6 5 0 E}+00(1.12 \mathrm{E}-01)$ \\
\hline PMOD $(\mathrm{k}=2.0)$ & $2.598 \mathrm{E}+01(1.11 \mathrm{E}+00)$ & $\mathbf{6 . 2 3 5 E + 0 0 ( 4 . 5 3 E - 0 2 )}$ & $2.884 \mathrm{E}+01(1.93 \mathrm{E}-01)$ & $\mathbf{1 . 7 8 3 E + 0 0 ( 3 . 6 3 E - 0 2})$ & $2.878 \mathrm{E}+01(2.33 \mathrm{E}-01)$ & $\mathbf{1 . 6 5 6 E + 0 0 ( 1 . 1 9 E - 0 1 )}$ \\
\hline
\end{tabular}

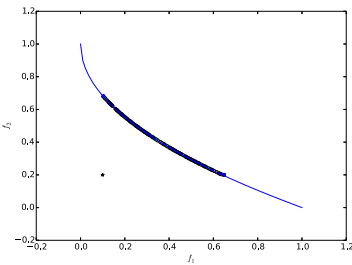

(a)g-NSGA-II

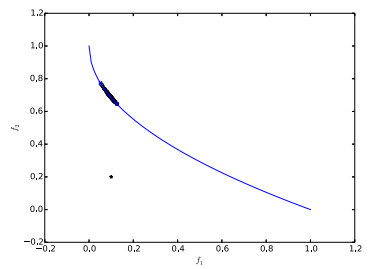

(b) r-NSGA-II

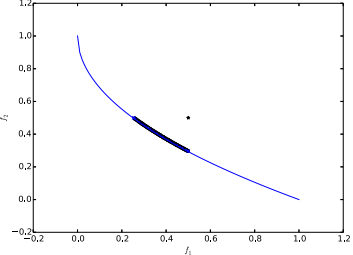

(b) g-NSGA-II

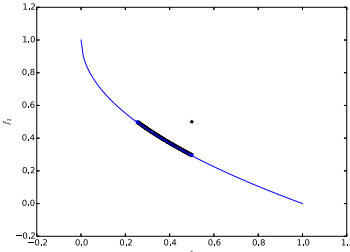

(b) r-NSGA-II

Fig. 6. Results on the sets of ZDT4 with reference points $(0.1,0.2)$ in infeasible region and $(0.5,0.5)$ in feasible.

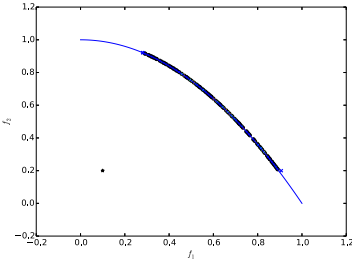

(a)g-NSGA-II

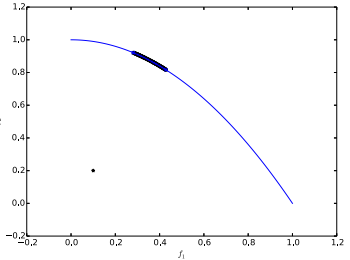

(b) r-NSGA-II

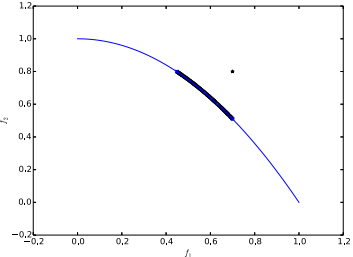

(b) g-NSGA-II

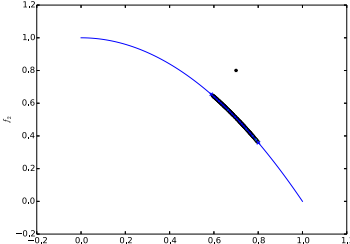

(b) r-NSGA-II

Fig. 7. Results on the sets of ZDT6 with reference points $(0.1,0.2)$ in infeasible region and $(0.7,0.8)$ in feasible region. 


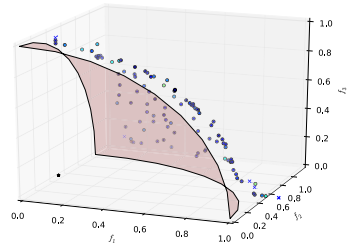

(a)g-NSGA-II

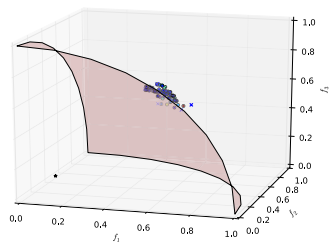

(b) r-NSGA-II

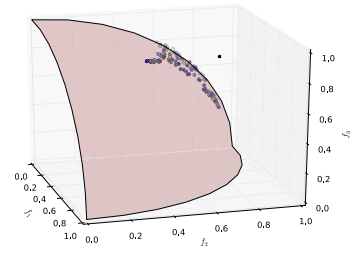

(b) g-NSGA-II

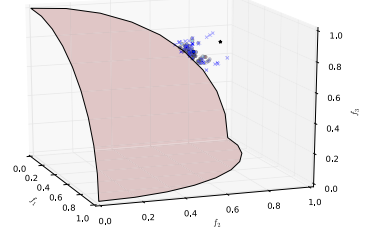

(b) r-NSGA-II

Fig. 8. Results on the sets of DTLZ2 with reference points $(0.1,0.2,0.1)$ in infeasible region and $(0.4,0.8,0.8)$ in feasible region.

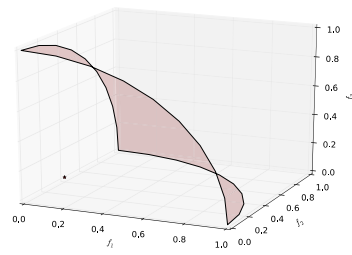

(a)g-NSGA-II

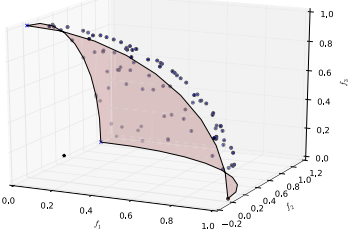

(b) r-NSGA-II

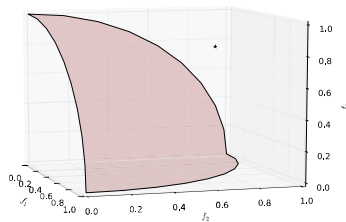

(b) g-NSGA-II

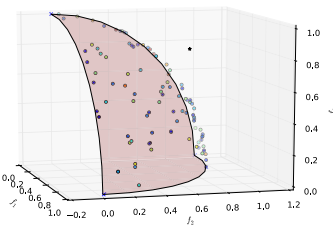

(b) r-NSGA-II

Fig. 9. Results on the sets of DTLZ3 with reference points $(0.1,0.2,0.1)$ in infeasible region and $(0.4,0.8,0.8)$ in feasible region.

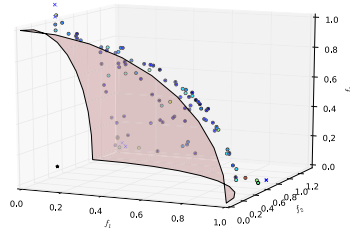

(a)g-NSGA-II

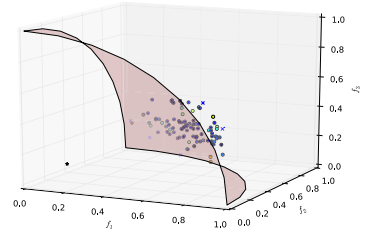

(b) r-NSGA-II

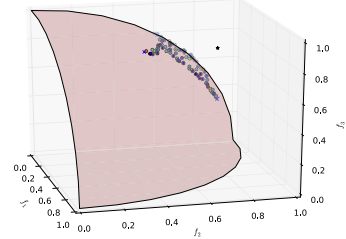

(b) g-NSGA-II

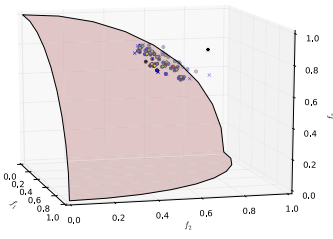

(b) r-NSGA-II

Fig. 10. Results on the sets of DTLZ4 with reference points $(0.1,0.2,0.1)$ in infeasible region and $(0.4,0.8,0.8)$ in feasible region.

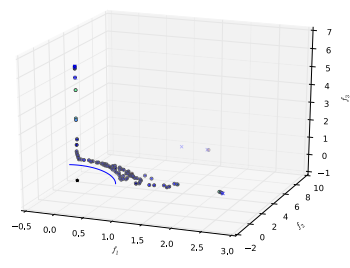

(a)g-NSGA-II

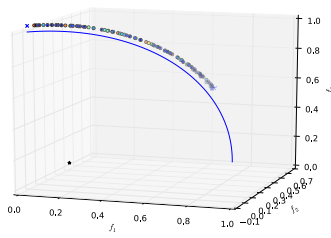

(b) r-NSGA-II

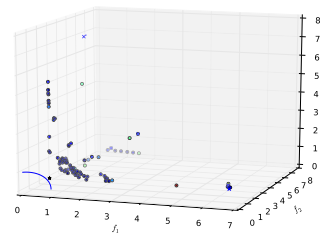

(b) g-NSGA-II

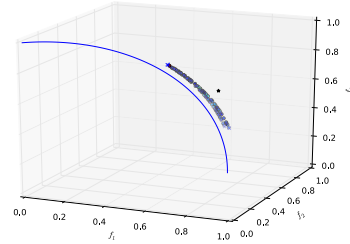

(b) r-NSGA-II

Fig. 11. Results on the sets of DTLZ6 with reference points $(0.1,0.2,0.1)$ in infeasible region and $(0.4,0.8,0.8)$ in feasible region. 


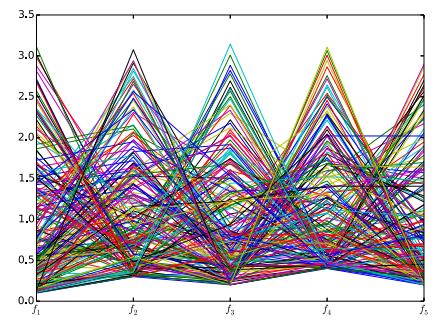

(a)g-NSGA-II

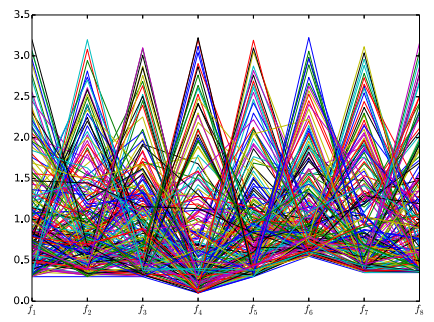

(b) g-NSGA-II

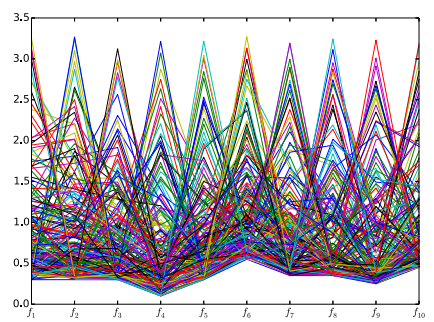

(b) g-NSGA-II

Fig. 12. Results on DTLZ2 with 5-,8-,10-objective by g-NSGA-II

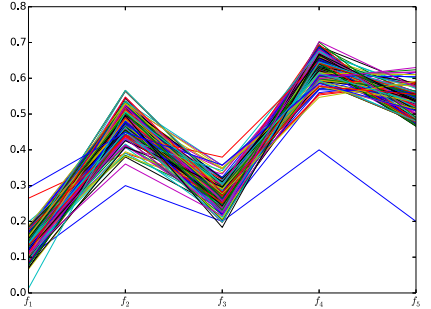

(a)r-NSGA-II

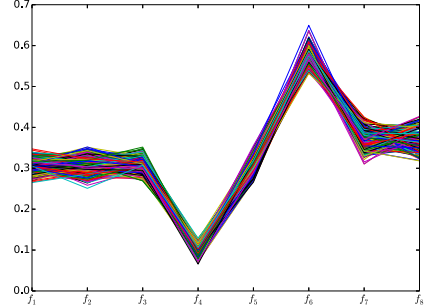

(b)r-NSGA-II

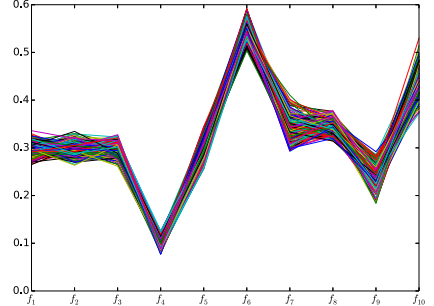

(b)r-NSGA-II

Fig. 13. Results on DTLZ2 with 5-,8-,10-objective by r-NSGA-II.

The DTLZ6 is a degenerate test problem which has a strong bias away from efficient front [21]. From Fig.11, we find that it creates a huge obstacle for g-NSGA-II to converge into the POF, and the DM is more satisfied with the solutions obtained by r-NSGA-II. To that point, the proposed performance indicator can give DM a reasonable value from table IV. Therefore, DM can choose a suitable algorithm to solve degenerate problems according to the indicator value.

In real-word applications, there are lots of problems with more than three objectives. According to Fig.12 and Fig.13, r-NSGA-II has a better performance than g-NSGA-II when dealing with many-objective problems. The effectiveness of the proposed indicator is confirmed by the analysis of the indicator value shown in Table $\mathrm{V}$.

\section{CONCLUSIONS}

Over the past decade, preference-based MOEAs have attracted increasing attention from both academia and industry due to their significance in both theory and practice. Learning how to assess the performance of preference-based MOEAs is a key issue that needs to be solved. The IGD-CF heavily depends on a composite front and requires the normalization of the objective space. The R-metric needs the knowledge of POF and demands DM to give additional information.

In this paper, we have presented a new performance indicator for preference-based MOEAs from a real-life perspective. It can measure the performance of preference-based algorithms through simple distances calculation. It is significant that the proposed indicator does not need to know the POF of benchmark problems and does not need to normalize the objective space.

However the indicator needs to provide an extra penalty coefficient $k$ and no detail proofs are provided that it actually conforms to the mathematical requirements of a metric. In addition, the indicator uses the uniformity of preferred solutions as a measure of diversity and does not take into account the spread of solutions, since preference-based MOEAs determine the size and shape of the ROI in different ways, and in several methods is hard to get a specific size of ROI. Therefore, in future work, we will investigate about what would be an ideal ROI in terms of size and boost the development of evaluating methods for preference-based evolutionary multiobjective optimization.

\section{ACKNOWLEDGMENT}

This work was supported by the research projects: the National Natural Science Foundation of China under Grant Nos. 61502408, 61673331, 61379062 and 61403326, the Education Department Major Project of Hunan Province under Grant No. 17A212, the CERNET Innovation Project under Grant No. NGII20150302, the Natural Science Foundation of Hunan Province under Grant No. 14JJ2072, the Science and Technology Plan Project of Hunan Province under Grant No. 2016TP1020, the Provinces and Cities Joint Foundation Project under Grant No. 2017JJ4001. 


\section{REFERENCES}

[1] R. Cheng, Y. Jin, M. Olhofer, B. Sendhoff, A reference vector guided evolutionary algorithm for many-objective optimization, IEEE Transactions on Evolutionary Computation 20 (5) (2016) 773-791.

[2] R. Cheng, T. Rodemann, M. Fischer, M. Olhofer, Y. Jin, Evolutionary many-objective optimization of hybrid electric vehicle control: from general optimization to preference articulation, IEEE Transactions on Emerging Topics in Computational Intelligence 1 (2) (2017) 97-111.

[3] J. Zou, Y. Zhang, S. Yang, Y. Liu, J. Zheng, Adaptive neighborhood selection for many-objective optimization problems, Applied Soft Computing.

[4] K. Li, K. Deb, Q. Zhang, S. Kwong, An evolutionary many-objective optimization algorithm based on dominance and decomposition, IEEE Transactions on Evolutionary Computation 19 (5) (2015) 694-716.

[5] G. Ruan, G. Yu, J. Zheng, J. Zou, S. Yang, The effect of diversity maintenance on prediction in dynamic multi-objective optimization, Applied Soft Computing 58 (2017) 631-647.

[6] J. Molina, L. V. Santana, A. G. Hernández-Díaz, C. A. C. Coello, R. Caballero, g-dominance: Reference point based dominance for multiobjective metaheuristics, European Journal of Operational Research 197 (2) (2009) 685-692.

[7] L. B. Said, S. Bechikh, K. Ghédira, The r-dominance: a new dominance relation for interactive evolutionary multicriteria decision making, IEEE Transactions on Evolutionary Computation 14 (5) (2010) 801-818.

[8] G. Yu, J. Zheng, R. Shen, M. Li, Decomposing the user-preference in multiobjective optimization, Soft Computing 20 (10) (2016) 4005-4021.

[9] S. F. Adra, I. Griffin, P. J. Fleming, A comparative study of progressive preference articulation techniques for multiobjective optimisation, in: International Conference on Evolutionary Multi-Criterion Optimization, Springer, 2007, pp. 908-921.

[10] K. Li, Q. Zhang, S. Kwong, M. Li, R. Wang, Stable matching-based selection in evolutionary multiobjective optimization, IEEE Transactions on Evolutionary Computation 18 (6) (2014) 909-923.

[11] J. Zheng, G. Yu, Q. Zhu, X. Li, J. Zou, On decomposition methods in interactive user-preference based optimization, Applied Soft Computing 52 (2017) 952-973.

[12] J. Hu, G. Yu, J. Zheng, J. Zou, A preference-based multi-objective evolutionary algorithm using preference selection radius, Soft Computing 21 (17) (2017) 5025-5051.

[13] V. G. da Fonseca, C. M. Fonseca, A. O. Hall, Inferential performance assessment of stochastic optimisers and the attainment function, in: International Conference on Evolutionary Multi-Criterion Optimization, Springer, 2001, pp. 213-225.

[14] E. Zitzler, L. Thiele, M. Laumanns, C. M. Fonseca, V. G. Da Fonseca, Performance assessment of multiobjective optimizers: an analysis and review, Evolutionary Computation, IEEE Transactions on 7 (2) (2003) $117-132$.

[15] A. Ibrahim, S. Rahnamayan, M. V. Martin, K. Deb, 3d-radvis antenna: Visualization and performance measure for many-objective optimization, Swarm and evolutionary computation 39 (2018) 157-176.

[16] A. Mohammadi, M. N. Omidvar, X. Li, A new performance metric for user-preference based multi-objective evolutionary algorithms, in: Evolutionary Computation (CEC), 2013 IEEE Congress on, IEEE, 2013, pp. 2825-2832.

[17] K. Li, K. Deb, Performance assessment for preference-based evolutionary multi-objective optimization using reference points, COIN Report 1 (1) (2016) 1-23.

[18] M. Li, J. Zheng, Spread assessment for evolutionary multi-objective optimization, in: International Conference on Evolutionary Multi-Criterion Optimization, Springer, 2009, pp. 216-230.

[19] K. Deb, Multi-objective genetic algorithms: Problem difficulties and construction of test problems, Evolutionary computation 7 (3) (1999) $205-230$.

[20] K. Deb, L. Thiele, M. Laumanns, E. Zitzler, Scalable test problems for evolutionary multiobjective optimization, Springer, 2005.

[21] Q. Zhang, H. Li, MOEA/D: A multiobjective evolutionary algorithm based on decomposition, IEEE Transactions on evolutionary computation, 11 (6) (2007) 712-731. 